\title{
Evaluation of the alleviative action of neurotropin for penile pain associated with intracavernous injection of prostaglandin E1 assessed using the visual analogue scale
}

\author{
Y Sato, N Suzuki, H Adachi, S Hisasue, H Horita and T Tsukamoto \\ Department of Urology, Sapporo Medical University School of Medicine
}

\begin{abstract}
We examined whether penile pain associated with intracavernous injection of PGE1 could be alleviated with a intracavernous injection of neurotropin, a non-protein, bioactive agent extracted from skin tissue with inflammation elicited by the Vaccinia virus.

Twenty-three patients with erectile dysfunction were enrolled in this study. They were divided into two groups. The first group was allocated $20 \mu \mathrm{g}$ of PGE1 alone first, followed by PGE1 plus $1.5 \mathrm{ml}$ of neurotropin after two weeks. The second group was allocated PGE1 plus $1.5 \mathrm{ml}$ of neurotropin first, followed by PGE1 alone after two weeks.

Pain was evaluated by the visual analogue scale (VAS). In the 17 patients reporting discomfort, pain scores for PGE1 injection with neurotropin were significantly lower than those without neurotropin. No patients showed local or systemic complications that might be related to neurotropin.

From these data we conclude that neurotropin may be an effective and safe drug for penile pain associated with PGE1 intracavernous injection.
\end{abstract}

Keywords: penile pain; visual analogue scale; PGE1

\section{Introduction}

Intracavernous injection of prostaglandin E1 (PGE1) is widely used in the evaluation and treatment of erectile dysfunction. ${ }^{1}$ However, the most common unfavorable side effect associated with usage of PGE1 is penile pain. The causes of PGE1-associated penile pain are not well known. Several studies indicated that the acidity or ionization state of the PGE1 solution, ${ }^{2}$ the alcohol-based formulation, ${ }^{3}$ the speed of injection ${ }^{4}$ and direct activation of a pain receptor ${ }^{5}$ may be related to the pain.

PGE1 enhances the pain and vascular permeability induced by bradykinin. ${ }^{6,7}$ Neurotropin inhibits the release of a bradykinin-like substance,$^{8}$ and is a non-protein, bioactive agent extracted from rabbit skin tissue with inflammation elicited by the Vaccinia virus. ${ }^{9}$ It has some biological actions, including, among others, analgesic action, antiallergic action, and immuno-potentiative action. ${ }^{10,11}$ Clinically, neurotropin inhibits the phlebitis in-

Correspondence: Dr Y Sato, Department of Urology, School of Medicine, Sapporo Medical University, S-1, W-16, Chuo-ku, Sapporo 060, Japan.

Received 4 March 1997; revised 10 June 1997; accepted 25 July 1997 duced by intravenous injection of PGE1. ${ }^{12}$ We preliminary reported that penile pain associated with PGE1 injection was alleviated by a concomitant injection of neurotropin. ${ }^{13}$ However, in the previous report, evaluation for pain was not sophitiscated because of use of a original pain score. Therefore, we examined whether the PGE1-induced pain could be alleviated with a concomitant intracavernous injection of neurotropin, using a more appropriate evaluation method, the visual analogue scale (VAS), ${ }^{14,15}$ VAS is considered to be a reliable and generalized method for evaluation of pain.

\section{Materials and methods}

Twenty-three patients with erectile dysfunction were enrolled in this study after informed consent was obtained. Their ages ranged from 29-71y (mean; 58.6 y). The study was conducted by dividing the 23 patients into two groups. The first group was allocated $20 \mu \mathrm{g}$ of PGE1 alone first, followed by PGE1 plus $1.5 \mathrm{ml}$ of neurotropin after two weeks. The second group was allocated PGE1 plus $1.5 \mathrm{ml}$ of neurotropin first, followed by PGE1 alone after two weeks. The patients were not informed of the order of administration of drugs. Therefore, this study was 
considered a blinded cross-over one. Twenty $\mu \mathrm{g}$ of PGE1 was dissolved with $1.5 \mathrm{ml}$ of saline or $1.5 \mathrm{ml}$ of neurotropin and their pHs at $27^{\circ} \mathrm{C}$ are 5.0 and 7.2 , respectively. PGE1 was injected at the unilateral corpus cavernosum with a $26 \mathrm{G}$ needle for $15 \mathrm{~s}$. The employed PGE1 was provided as a freeze-dried powder containing $20 \mu \mathrm{g}$ of alprostadil and lactose as a stabilizer (Ono Pharmaceutical Co., Ltd. Tokyo, Japan). The solution has a pH of 3.5-5.5. Neurotropin (Nippon Zoki Co., Ltd. Tokyo, Japan) is a nonprotein, bioactive agent extracted from inflamed rabbit skin inoculated with vaccinia virus. It contains peptides, polysaccharides, nucleic acid, amino acid and its molecular volume is less than $2300^{9}$. The neurotropin used contained $1.2 \mathrm{mg}$ of extract per $1 \mathrm{ml}$ of solution with a $\mathrm{pH}$ of 7.0-8.0.

We observed erectile response after injection for $20 \mathrm{~min}$. Erectile response was evaluated at $20 \mathrm{~min}$ after injection and categorized as, good, fair, or poor. After observation of the erectile response, all patients were required to indicate a degree of their pain on the visual analogue scale (VAS). ${ }^{14,15}$ The pain scores were calculated as the length $(\mathrm{cm})$ from 0 to the checked point. Data were analyzed by paired $t$-test.

\section{Results}

We excluded six patients who felt no pain at both types of injections from analysis. Eight patients in the first group and nine patients in the second group reported some degree of pain when PGE1 was injected with or with neurotropin. In both groups, pain scores of PGE1 injection with neurotropin were significantly lower than those without neurotropin $(P<0.01)$. Regardless of the order of administration, neurotropin significantly alleviated the PGE1-associated penile pain (Table 1). No difference was recognized between individual erectile responses with or without neurotropin except by one patient in the first group. His poor response to the initial injection without neurotropin changed to a fair response to the second injection with neurotropin. No patients showed local or systemic complications that might be related to neurotropin.

Table 1 Pain scores with PGE1 injection with or without neurotropin

\begin{tabular}{lcccc}
\hline & \multicolumn{4}{c}{ Pain scores } \\
\cline { 2 - 5 } & $\begin{array}{c}\text { PGE1+ } \\
\text { saline }\end{array}$ & & $\begin{array}{c}\text { PGE1 }+ \\
\text { Neurotropine }\end{array}$ \\
\hline First group $(n=8)$ & $4.2 \pm 1.8$ & vs & $2.3 \pm 1.9$ & $P<0.01$ \\
Second group $(n=9)$ & $4.2 \pm 2.2$ & vs & $2.4 \pm 1.7$ & $P<0.01$ \\
Total $(n=17)$ & $4.2 \pm 2.0$ & vs & $2.3 \pm 1.8$ & $P<0.01$ \\
\hline
\end{tabular}

\section{Discussion}

The most unfavorable side effect of intracavernous injection of PGE1 is penile pain. The incidence of penile pain had been reported to be from $10 \%$ to $80 \% .^{2-4}$ Porst reviewed the literature and found the incidence of penile pain in self injection to be $7.2 \%{ }^{1}$ Thus, such pain has not been the main reason for dropping out of self-injection programs using PGE1. ${ }^{1}$ However, if the pain could be controlled, the number of candidates for self-injection with PGE1 would be increased.

In this study, we employed the VAS for the evaluation of penile pain. The VAS is considered to be a reliable method for this purpose. ${ }^{14,15}$ Regardless of the order of administration, neurotropin significantly alleviated the PGE1-associated penile pain.

Neurotropin has analgesic action, anti-allergic action and immuno-potentiative action. ${ }^{10,11}$ It is used for treatment of lumbago and neuralgia. However, its mechanism of pain reduction is not well understood, though there are some possible explanations. It has been reported that PGE1 enhances the pain induced by bradykinin. ${ }^{6,7}$ Imai et $a l^{8}$ indicated that neurotropin inhibited release of bradykinin-like substances evoked by noxious stimuli produced by pinching the paw skin of the rat. Therefore, we speculated that the mechanism of neurotropin alleviation of PGE1-associated penile pain might be related to its inhibitory action on the release of bradykinin-like substances. This hypothesis might be supported by the results of a clinical study, showing that neurotropin prevents phlebitis associated with intravenous injection of PGE1. ${ }^{9}$ Neurotropin has an anti-nociceptive action, which might be induced through the modulation of monoaminergic and GABAergic system functions. ${ }^{10,16}$ These effects also might contribute to alleviation of penile pain.

The $\mathrm{pH}$ of the drug solution might affect the penile pain induced by its injection. ${ }^{17}$ The $\mathrm{pH}$ of PGE1 dissolved with neurotropin was higher than with saline. Therefore, the difference of $\mathrm{pH}$ might contribute to the alleviative action of neurotropin for penile pain.

Neurotropin did not affect the erectile response induced by intracavernous injection of PGE1. No patients showed local or systemic complications, and there are no reports of significant complications due to administration of neurotropin.

\section{Conclusion}

Our results indicated that neurotropin could alleviate pain caused by intracavernous injection of PGE1 without complications. Neurotropin may be an effective and safe drug for penile pain associated 
with PGE1 intracavernous injection. Double blind study will be necessary for confirming our result.

\section{References}

1 Porst H. The rationale for prostaglandin E1 in erectile failure: a survey of worldwide experience. J Urol 1996; 155: 802-815.

2 Moriel EZ, Rajfer J. Sodium bicarbonate alleviates penile pain induced by intracavernous injections for erectile dysfunction. J Urol 1993; 149: 1299-1300.

3 Chen J, Godschalk MK, Katz PG, Mulligan T. Incidence of penile pain after injection of new formulation of Prostaglandin E1. J Urol 1995; 154: 77-79.

4 Gheorghiu D, Godschalk M, Gheorghiu D, Mulligan T. Slow injection of prostaglandin E1 decreases associated penile pain. Urology 1996; 47: 903-904.

5 Schramek P, Plas EG, Hübner WA, Pflüger H. Intracavernous injection of prostaglandin E1 plus procaine in the treatment of erectile dysfunction. J Urol 1994; 152: 1108-1110.

6 Horton EW. Action of prostaglandin E1 on tissues which respond to bradykinin. Nature 1963; 200: 892.

7 Ferreira SH. Prostaglandins, aspirin-like drugs and analgesia. Nature New Biology 1972; 240: 200-203.
8 Imai Y, et al. Inhibition of the release of bradykinin-like substances into the perfusate of rat hind paw by neurotropin. Japan J Pharmacol 1984; 36: 104-106.

9 Takino M. Patent publication in Japan 1970; NO.27996.

10 Hata T et al. Mechanism of the analgesic effect of neurotropin. Japan J Pharmacol 1988; 48: 165-173.

11 Takeiti N, Ba D, Koga Y, Kobayashi H. Immunologic suppression of carcinogenesis in spontaneously hypertensive rats (SHR) with T cell depression. J Immunol 1983; 130: 501-505.

12 Ogihara $\mathrm{M}$ et al. A clinical and experimental study on effect of neurotropin for phlebitis due to Prostaglandin E1. The Clinical Report 1992; 26: 4541-4543.

13 Suzuki N et al. Effect of neurotropin for penile pain induced by intracavernous injection of prostaglandin E1. Impotence 1996; 11: 25-28.

14 Aitken RCB: Measurement of feeling using visual analogue scales. Proc Roy Soc Med 1969; 62: 989-993.

15 Price DD, McGrath PA, Rafii A, Buckingham B. The validation of visual analogue scales as ratio scale measures for chronic and experimental pain. Pain 1983; 17: 45-56.

16 Ohara $\mathrm{H}$ et al. Mechanism of hyperalgesia in SART stressed (repeated cold stress) mice: antinociceptive effect of neurotropin. Japan J Pharmacol 1991; 57: 243-250.

17 Moriel EZ, Rajfer J. Sodium bicarbonate alliviates penile pain induced by intracavernous injections for erectile dysfunction. J Urol 1993; 149: 1299-1300. 